\title{
DEVELOPING THE STABILIZED MAPPING SYSTEM FOR THE GYROCOPTER - REPORT FROM THE FIRST TESTS
}

\author{
J. Kolecki ${ }^{\text {a, } *, \text { M. Prochaska }}{ }^{\text {a }}$ Z. Kurczyński ${ }^{\text {a }}$, P. Piątek ${ }^{\text {b }}$, J. Baranowski ${ }^{\text {c }}$ \\ a ADRAM Ltd. Zabierzów, Poland (kkolecki, mprochaska)@adram.pl, kurczynski@wp.pl \\ b piatek.pawel@gmail.com \\ c jerzybaranowski@gmail.com
}

Commission I, WG I/2

KEY WORDS: autogyro, stabilization, LiDAR data quality, trajectory estimation, power spectral density

\begin{abstract}
:
The LiDAR mapping carried out using gyrocopters provides a relatively cheap alternative for traditional mapping involving airplanes. The costs of the fuel and the overall maintenance are much lower when compared to planes. At the same time the flight kinematics of the gyrocopter makes it an ideal vehicle for corridor mapping. However a limited payload and a strongly limited space prevent using stabilized platforms dedicated for aerial photogrammetry. As the proper stabilization of the laser scanner during the flight is crucial in order to keep the desirable quality of the LiDAR data, it was decided to develop the prototype of the stabilized, ultra-light mapping platform that can meet the restricted requirements of the gyrocopter. The paper starts with the brief discussion of the legal and practical aspects of the LiDAR data quality, dealing mostly with the influence of the flight imperfections on the point pattern and point density. Afterwards the mapping system prototype is characterized, taking into account three main components: stabilized platform, sensors and control. Subsequently first in-flight tests are described. Though the data are still not perfect mostly due to vibrations, the stabilization provides a substantial improvement of their geometry, reducing both roll and pitch deflections.
\end{abstract}

\section{INTRODUCTION}

\subsection{LiDAR data quality}

The aerial LiDAR measurements are currently widely applied in the acquisition of data for digital terrain modelling, generation of 3D city models, corridor mapping and other large-scale projects. In Poland the considerable part of the aerial laser scanning market is strictly related to government or local administration. To provide the overall rules of data acquisitions and to establish data standards, proper regulations had to be enacted by Polish Ministry of Interior and Administration in 2011 (MSWiA, 2011). Additionally each project, either commercial or governmental has its Terms of Reference or other kind of technical requirements, usually strictly defining data quality parameters and evaluation procedure.

The accuracy of the LiDAR measurements required for the most projects has generally become better than acceptable well time ago. In addition to demands concerning accuracy other restrictions involving point distribution are also significant. Among the recent LiDAR measurement campaigns that took place in Poland, the one carried out within the IT system of the country's protection against extreme hazards (pol. ISOK) has supplied the largest quantity of data. Terms of Reference prepared by National Water Management Authority in 2012 specify a bulk of conditions that must be fulfilled by LiDAR data. The restrictions concerning strip geometry and point distribution are as follows (Kurczyński and Bakuła, 2013):

side overlaps between strips: $\geq 20 \%$ of strip width and at least $100 \mathrm{~m}$,
- $\quad$ point density: $\geq 4 \mathrm{pts} / \mathrm{m}^{2}$ in rural and $\geq 12 \mathrm{pts} / \mathrm{m}^{2}$ in urban areas,

- the ratio of scan line distance and the distance between two subsequent points must be between $2 / 3$ and $3 / 2$.

The aforementioned quantities provide some idea about current standards and clearly show that the quality consists not only of the accuracy. The main sources of disturbances in the geometry of the LiDAR data are related to flight instability. This issue could be not so challenging for typical aerial mapping involving stabilized, gimbal platforms. However currently more and more data is acquired using ultralight airborne vehicles among which gyrocopters seem to provide quite a promising capabilities.

\subsection{Acquisition of images and LiDAR data using gyrocopter}

The gyrocopter, known also as autogyro (Fig. 1), is a lightweight vehicle that develops lift using rotor. The rotor is unpowered and rotates as a consequence of autorotation. The powered propeller provides the horizontal component of motion.

Photogrammetric surveys using gyrocopters are recently becoming more and more popular. Few mapping platforms of different costs and complexity have already been developed and used in practice (Adamek, 2014; Miraliakbari, 2010; Petrie 2014). A gyrocopter presents few features that are noteworthy for photogrammetric market. An autogyro is a lightweight and very compact vehicle. It can be transported to a take-off location using a car and a trailer. The costs of the fuel are insignificant when compared to plane. The maintenance is also

\footnotetext{
* Corresponding author
} 
much cheaper. Finally the costs of purchase are much lower than in case of planes or helicopters. Compared to plane, a gyrocopter can perform a safe flight slower, what is important when using cameras that are by default not equipped with the FMC (e.g. medium format cameras). Usually speed within the range of $70-100 \mathrm{~km} / \mathrm{h}$ is considered safe, but occasionally the gyrocopter can fly slower or may even come to a complete stop almost like a helicopter. Slower flight allows obtaining higher forward overlap of images and higher LiDAR data density. At the same time the overall flight duration can be much longer than in case of UAVs and as a result a much larger area can be covered by data. Besides the turn radius of the autogyro is small enough to perform the corridor mapping without wasting time for large-radius turns.

On the other hand conducting laser scanning using gyrocopter is not free from problems. Because of the limited payload and space it is impossible to mount the fully equipped mapping system with the gimbal and large format camera onboard. As a result of crosswind the gyrocopter often flights with considerable crab heading, imposing negative impact especially on side and on forward image overlap ratios. Considerable decrease of both LiDAR and imagery data quality can be caused by vibrations generated by engine and rotor. Miraliakbari et al present an extensive study of this problem (Miraliakbari et al, 2012). The presence of high roll, pitch and yaw motion rates resulting from wind blasts and convection streams rise the problem of sensor stabilization to the range of insurmountable challenge.

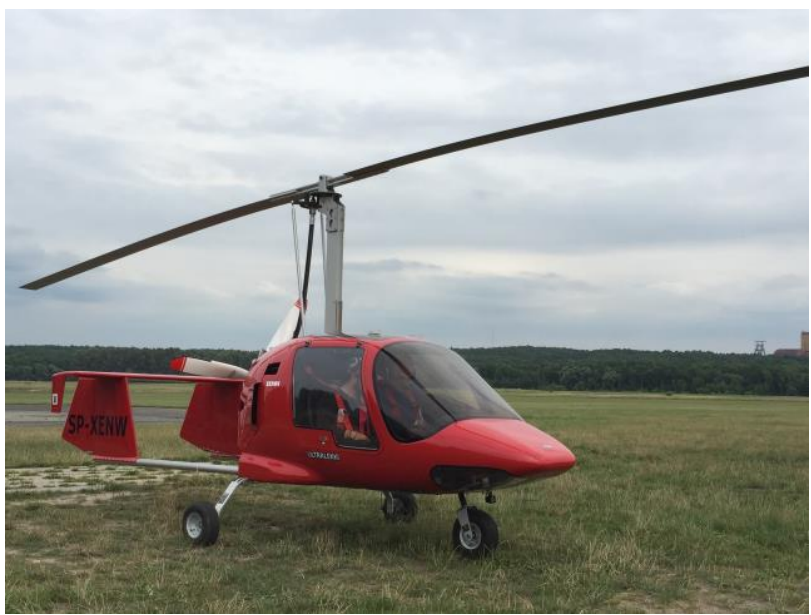

Figure 1. Gyrocopter.

To benefit from all the possibilities the gyrocopter provides and not to be stuck in the problematic trade-off between economy and data quality it was decided to develop the lightweight mapping system with the high quality sensors and stabilization mount. Using such a system it would be possible to acquire LiDAR data of quality comparable to aerial systems.

The paper deal with first tests of the developed stabilized system conducted in flight conditions. The main goal of the tests was to assess the performance of the stabilization. Besides the tests allowed to check the completeness of data registration and examine the accuracies of the estimates of the trajectory parameters.

\section{MAPPING SYSTEM ARCHITECTURE}

\subsection{Sensors}

The mapping platform prototype is equipped with the following sensors:

\section{Applanix POS AV OEM GNSS/INS system, Riegl VQ-580 laser scanner, \\ Phase One iXA 180 medium format camera.}

The Applanix system acquires trajectory data providing direct georeferencing for the mapping data. The GNSS receiver uses the antenna that is mounted outside. During the first tests the antenna was mounted on the roof of the autogyro.

Riegl laser scanner is mounted directly beneath the IMU (Fig. 2, Fig. 3). The VQ-580 system allows scanning with $380 \mathrm{KHz}$ frequency (respecting proper multiple time around zone) with the beam footprint of $22 \mathrm{~mm} @ 100 \mathrm{~m}$ and the field of view up to $60^{\circ}$ (Reigl, 2013). The medium format PhaseOne iXA 180 camera, that is mounted aside the scanner provides acquisition of $80 \mathrm{MPx}$ images in the up to $1.8 \mathrm{~s}$ interval. Using $55 \mathrm{~mm}$ lens results in $3 \mathrm{~cm}$ pixel @ $317 \mathrm{~m}$ altitude.

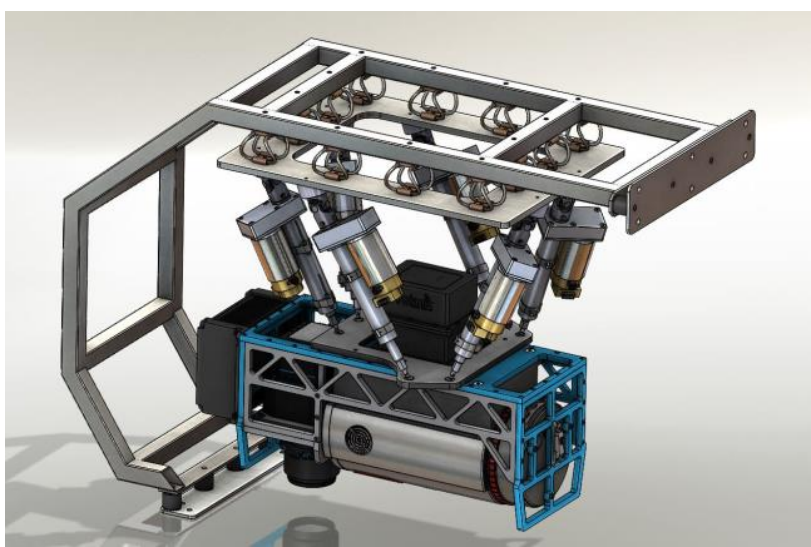

Figure 2. Mapping platform prototype: 3D CAD model.

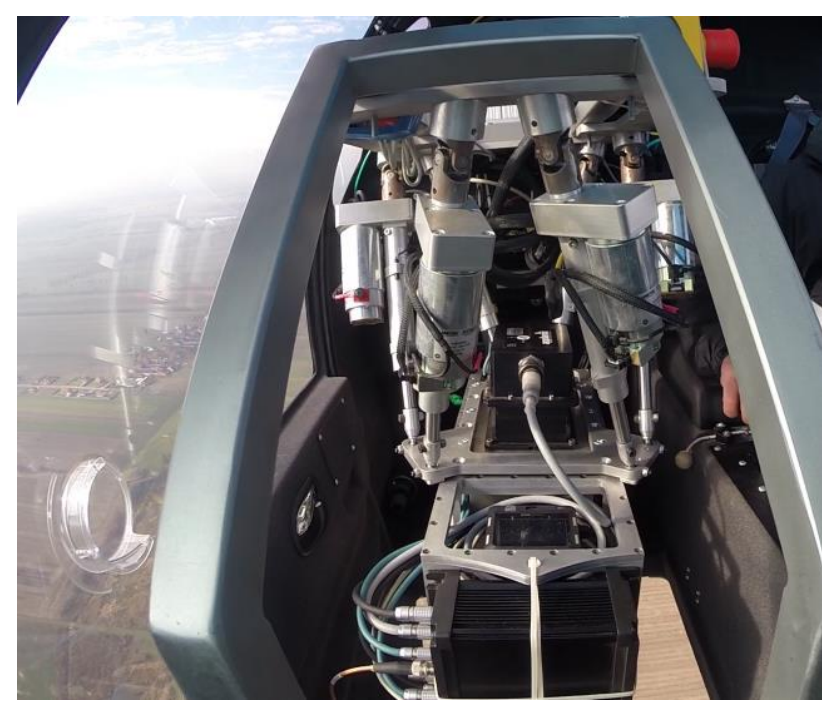

Figure 3. Mapping platform prototype during the flight. Image from the GoPro camera. 


\subsection{Stabilization mount}

For the stabilization of a measurement system a parallel robot in the Stewart's platform was used (Gough \& Whitehall, 1962; Stewart, 1965). The choice of such mechanical construction over traditionally used Gimbal systems was dictated by two main reasons. The first one comes from the high configurability and relatively small size of the platform. They allow scalability and adaptation to the small load capacities of the gyrocopter. The other advantage of the platform comes from the possibility of changing the center of rotation with control algorithm. In case of gimbal constructions the center of rotation is fixed with mechanical constraints. The benefit is that the center of rotation can be moved to the scanner or to the camera with a software decision, depending on the current needs. However, those merits come with certain costs. One of the problems comes from difficulties in control algorithms, as the direct kinematics of the parallel robots are generally only numerically solvable. The other problem is the difficulty in the determination of the robot position, as it is difficult to measure angles at pivot joints.

\subsection{Control}

The mapping platform is fitted with necessary control interface allowing data acquisition in the one-man mission mode. The flight is carried out according to the plan. The system computer using the dedicated navigation software gathers the navigation data from the Applanix and triggers the laser scanner at the beginning of the flight lines. Pilot navigates according to a flight plan displayed at the touch screen. Pilot can switch between flight lines and verify the data completeness online. The GUI of flight control application was developed with the cooperation with pilots and is assumed to distract only the minimum of operator attention.

The camera is triggered as the gyrocopter approaches the planned projection center. At the same time the computer records the INS/GNSS data stream, LiDAR raw data and image files. The stabilization mount control uses the on-line estimates of roll, pitch and possibly yaw values provided by the AHRS rigidly mounted inside the cockpit of the autogyro. The AHRS is roughly aligned with the body frame of the gyrocopter. During the tests the Xsens MTi ARHS was used for this purpose. Heading information was assumed unreliable as it comes mainly from the magnetometers. As a result it was decided not to stabilize the yaw motion during the test flights. In the future the application of more reliable yaw source, like two-antenna GPS receiver combined with magnetometers and MEMS gyro, is going to be taken under consideration. The attitude provided by the AHRS is passed to the Stewart Platform control in order to perform stabilization. When the stabilization works, the antenna reference point moves with respect to the frames of mapping sensors. It is therefore necessary to pass corrections to the Applanix system to allow compensating for antenna relative movement. A Stewart Platform motion is assumed to imitate the behavior of the gimbal - the motion is constrained to rotation only. This allows streaming the orientation information from the platform controller to the Applanix system in the format that is the same as the format of one of the supported gimbals.

\section{TESTS}

The very first test flights aimed to verify how the stabilization mount performs in the real flight condition. After overcoming some problems with communication it was finally possible to conduct the flights. Two flights were assumed at least partly successful (Fig. 4, Fig. 5). Both took place along 4 coinciding fling lines. The length of each line was about $3000 \mathrm{~m}$. The altitude was about $500 \mathrm{~m}$. The speed depended on the flight direction with respect to wind and ranged between 70 and 120 $\mathrm{km} / \mathrm{h}$. While flying along lines 1 and 2 in each flight the stabilization mount was turned on, and the Stewart platform was compensating roll and pitch motion of the aircraft. While flying along lines 3 and 4 the stabilization mount was deactivated with the aim of comparison. The LiDAR data were recorded for each line.

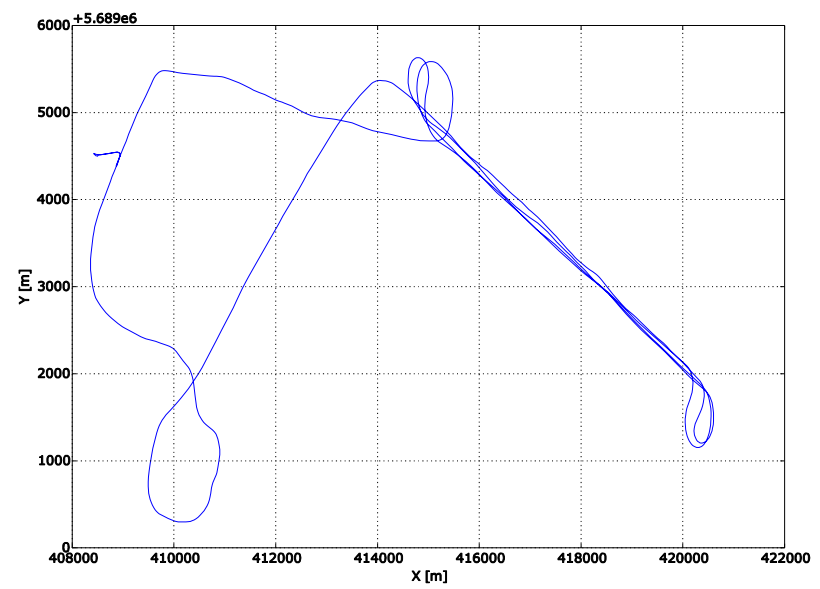

Figure 4. Trajectory line in top view. Flight \#1.

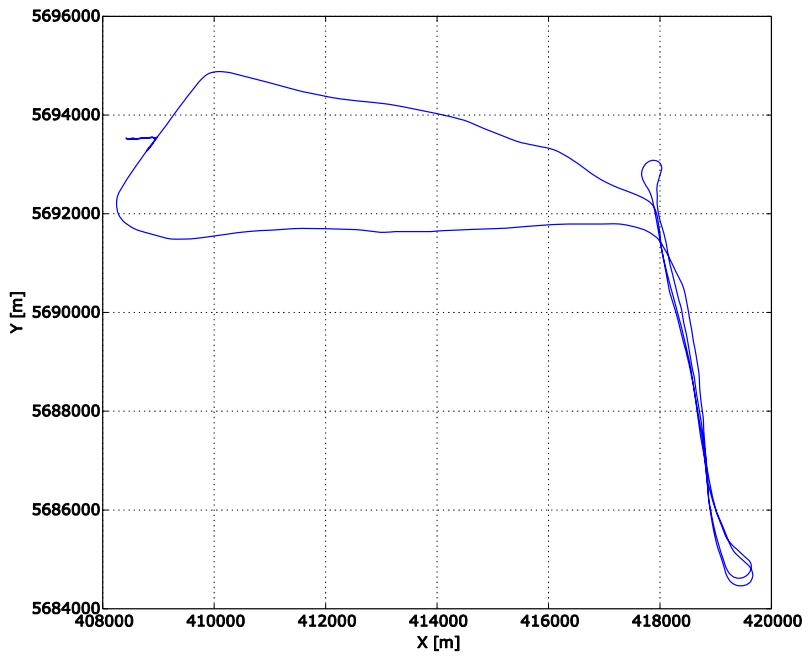

Figure 5. Trajectory line in top view. Flight \#2.

\section{RESULTS}

\subsection{Trajectory data evaluation}

After accomplishing the test flights the trajectory data recorded by the Applanix system were processed using corrections from GNSS reference station. Unfortunately there was a problem in acquisition of GPS signal during the second flight, so that narrow integer solution was unavailable. The estimates of coordinate accuracies reached few meters. As a consequence it was impossible to georeference LiDAR data captured during this flight. The estimates of the standard deviations of the coordinates of the first flight appear much better. They are provided in the figure 6 . Figure 7 shows the estimates of the 
accuracies for angular values. Grey strips feature 4 flight lines. For roll, pitch and yaw accuracies (Fig. 7) a narrower time period is shown for clarity. A considerable accuracy drop is visible starting from strip 3 , especially for the $\mathrm{Z}$ coordinate (Fig. 6). Probably visibility to some satellites was lost while the gyrocopter was turning. Very poor accuracy measures obtained for coordinates within strip 3 and 4 disable proper georeferencing of LiDAR data. Standard deviations obtained for roll and pitch almost coincide, while the accuracy of yaw is about 3 time worse.

Figure 8 shows roll and pitch values estimated for the time interval comprising flying along lies 2 and 3. For line 2 the stabilization was turned on. As a result the roll and pitch values only few times exceeded $\pm 2^{\circ}$, in average keeping close to $0^{\circ}$. While flying the third line roll/pitch motion compensation was turned off. The time interval needed to complete this line was much longer because of opposite wind. Roll and pitch values are within the limits of 0 and 6 degrees. For both lines we can observe that rapid angular motions are present. However when the stabilization is turned off the amplitude of this movements is higher.

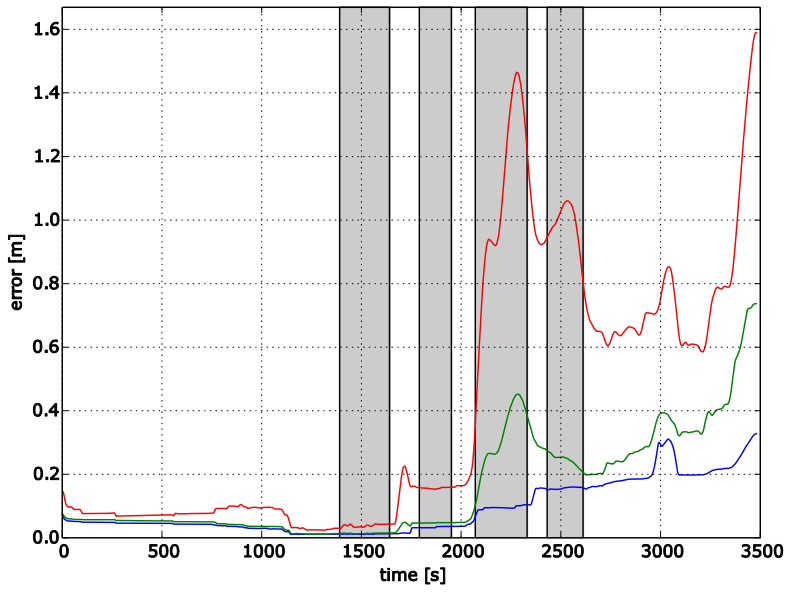

Figure 6. Estimated errors of X (blue), Y (green) and Z (red) coordinates, provided as a result of trajectory post-processing.

Flight \#1. Strips are marked by gray segments.

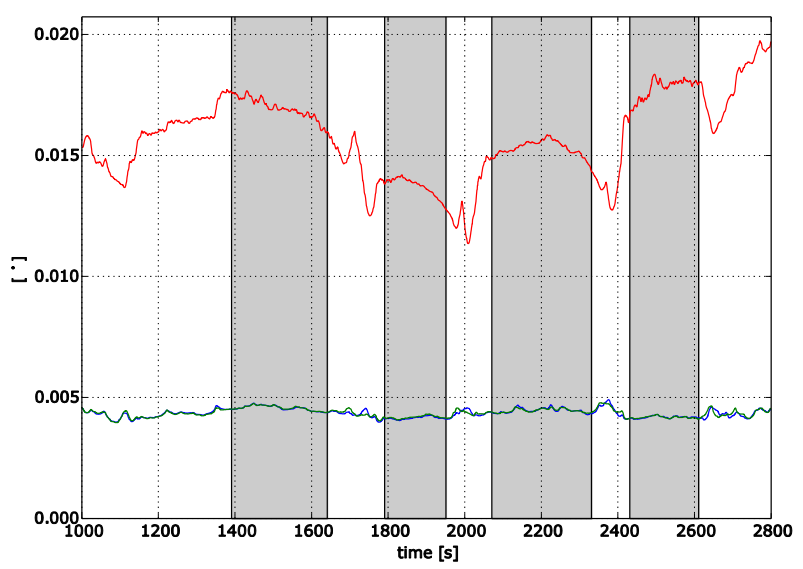

Figure 7. Estimated errors of roll (blue), pitch (green) and yaw (red) angles, provided as a result of trajectory post-processing.

Flight \#1. Strips are marked by gray segments. Data provided for period $1000-2800 \mathrm{~s}$.

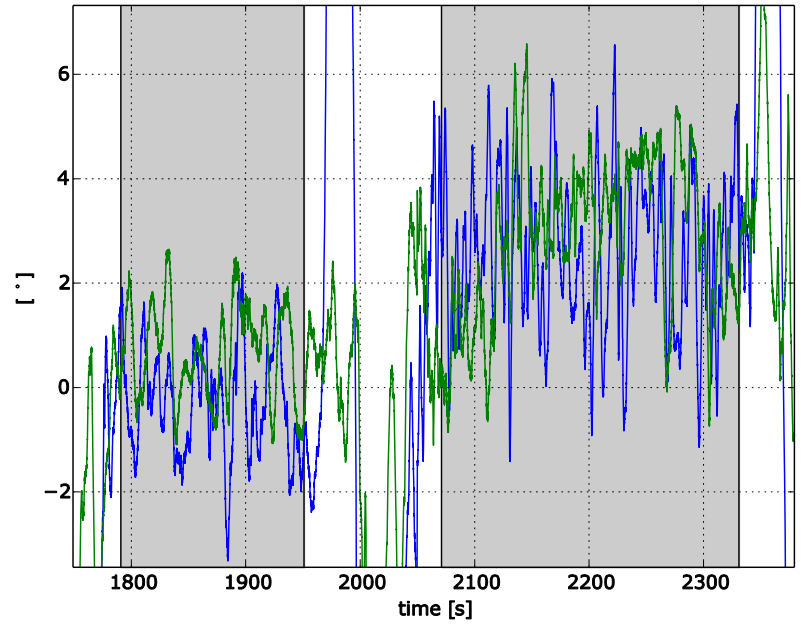

Figure 8. Roll (blue) and pitch (green) values for strip \#2 (with stabilization) and strip \#3 (no stabilization). Flight \#1.

Based on the post-processed trajectory data the cumulative distribution functions for roll and pitch values were calculated for both flights (Fig. 9, Fig.10, Fig. 11, Fig. 12). The calculation was carried out for absolute values, only for data recorded for strips. If the stabilization was active, at least $90 \%$ of values was below $2^{\circ}$, both for roll and pitch. When the stabilization was switched off the result were much worse.

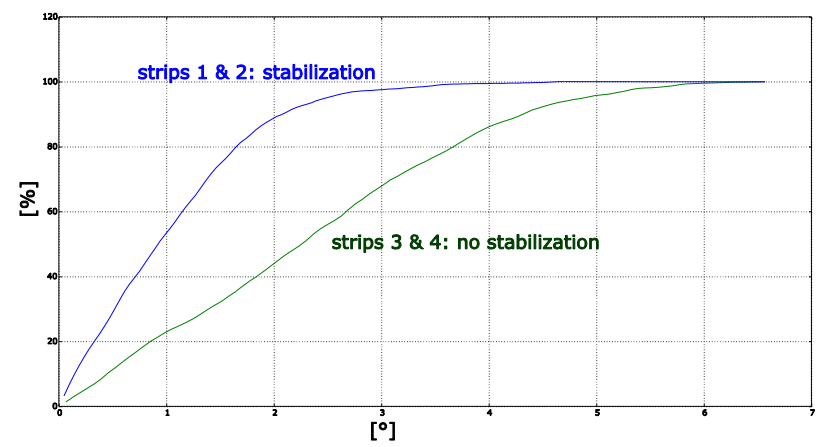

Figure 9. Empirical cumulative distribution function for roll values. Flight \#1.

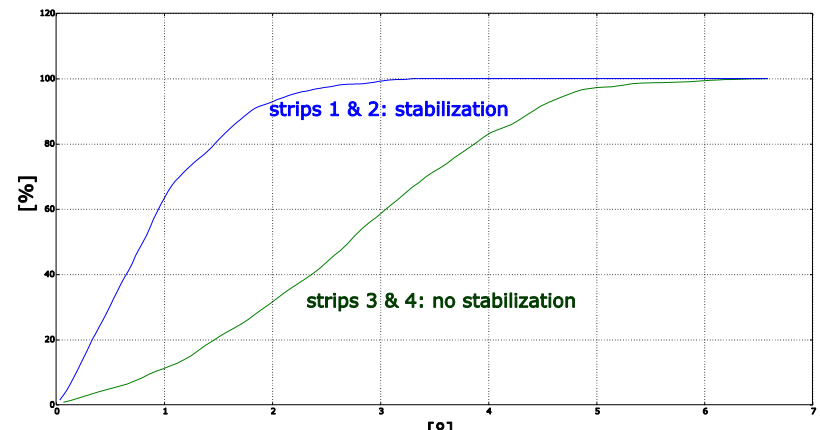

Figure 10. Empirical cumulative distribution function for pitch values. Flight \#1. 


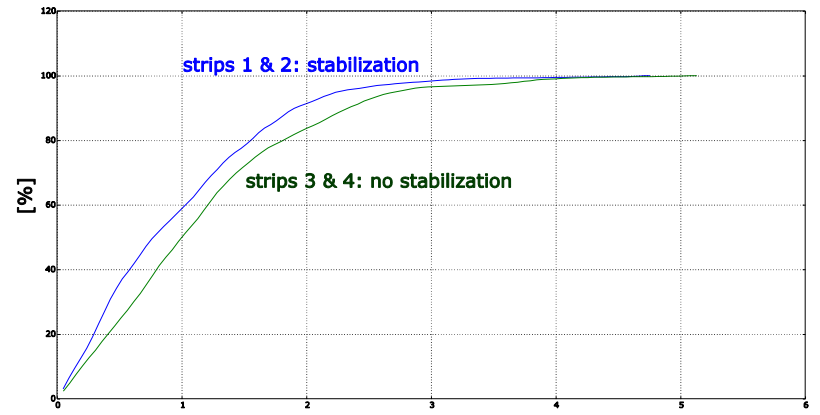

$\left[{ }^{\circ}\right]$

Figure 11. Empirical cumulative distribution function for roll values. Flight \#2.

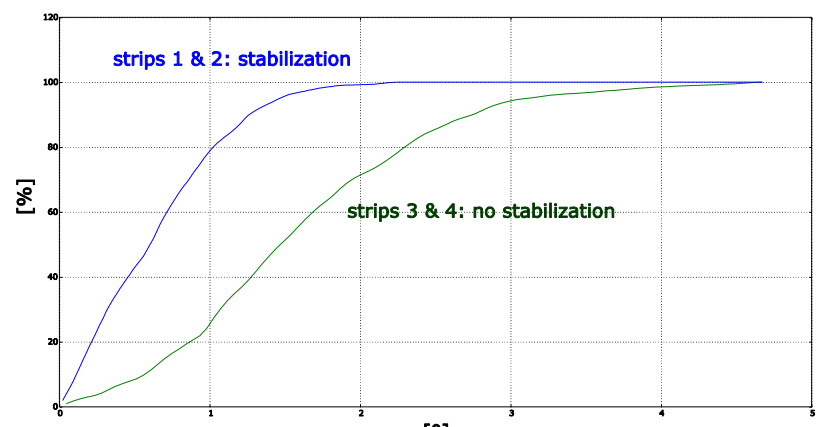

$\left[{ }^{\circ}\right]$

Figure 12. Empirical cumulative distribution function for pitch values. Flight \#2.

\subsection{Spectral analysis}

In order to assess the stabilization system orientation the frequency analysis methods were used. In order to do so the power spectral density (PSD) of the measured roll and pitch angles of the stabilized system. Power spectral density describes the frequency distribution of signal power and is used for frequency analysis of random signals (Zieliński, 2005). It is defined as a Fourier transform of autocorrelation function.

When analyzing the measurements of orientation of the platform in the gyrocopter we come across two influences. The first one are the periodic components coming from rotating and vibrating elements on the gyrocopter and the second come from random movements of the gyrocopter (caused by the weather and flight conditions, flight plan and pilot's skill). For computation of PSD instead of the popular periodogram the Welch's method was used (Zieliński, 2005; Stranneby, 2001). In most basic terms the Welch's method averages over several modified periodograms. It allows reduction of PSD dependence on signal length and its occurrence. Moreover additional advantage over periodogram is in that it reduces noise in the estimated power spectra in exchange for reducing the frequency resolution.

In figures 13 and 14 we presented PSD plots for roll and pitch angles respectively. Signals were obtained for flights with and without stabilization. As it can be observed stabilization reduced the inclinations of the platform for frequencies below $1 \mathrm{~Hz}$. It is a beneficial effect of the stabilization. For frequencies above $10 \mathrm{~Hz}$ the spectra are qualitatively identical. It means that the stabilization system does not operate in such frequency range. Applied electromechanical drives are to slow to compensate for such fast movement. The frequency range between 1 and $10 \mathrm{~Hz}$ shows that the inclinations in the stabilized flights are higher than in those without stabilization. This effect is obviously disadvantageous. The sources of this effect are probably errors in the stabilization controller tuning. In further works it will be attempted to improve control quality for this frequency range.

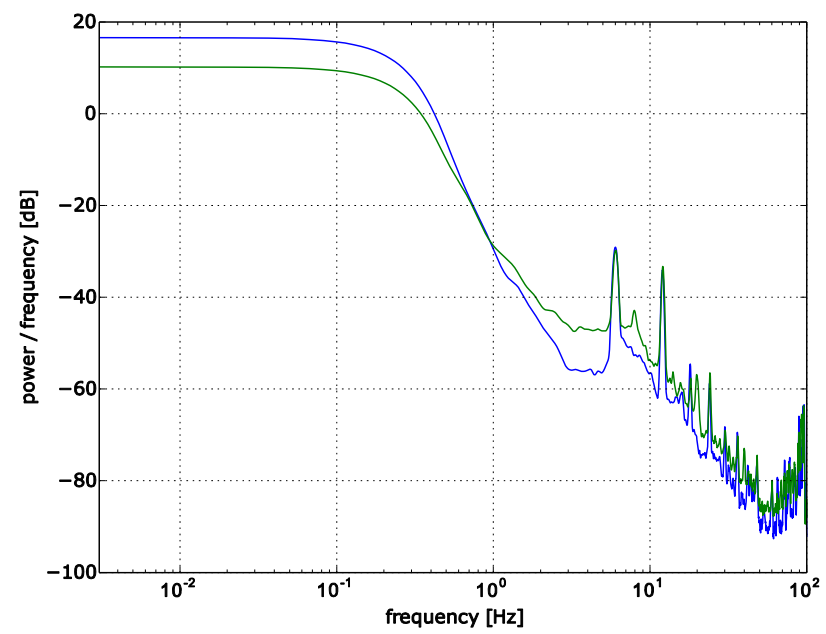

Figure 13. Power spectral density of the roll angle - Welch approach. Flight lines with stabilization - green, flight lines without stabilization - blue. Flight \#1.

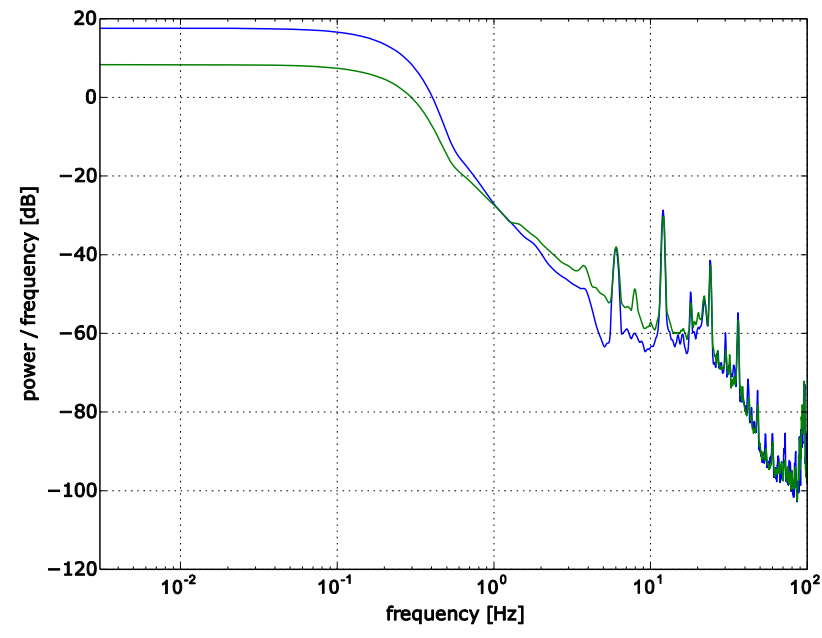

Figure 14. Power spectral density of the pitch angle - Welch approach. Flight lines with stabilization - green, flight lines without stabilization - blue. Flight \#2

\section{CONCLUSIONS}

Conducted tests show that in general the stabilization system allows to supress the low frequency components of roll and pitch motion of the platform. However the spectral density diagrams show that applying the stabilization increase the higher frequency components. The in-depth analysis of the impact of the stabilization on LiDAR data parameters was beyond the scope of the presented tests, but one can expect that applying the stabilization can significantly reduce the planned strip overlap required to guarantee the complete coverage of the mission area. However the presence of vibrations must have the disturbing impact on laser point pattern. One can consider improving the vibration insulation system to overcome the above mentioned problem. 
Most of the roll and pitch data samples recorded while the stabilization was active were within the limits of $\pm 2^{\circ}$ (see Fig. 8 - Fig.12). The $\pm 2^{\circ}$ corresponds to the accuracy of the Xsens MTi unit expected in the dynamic conditions. Currently MEMS AHRS units of one order of magnitude better accuracy are available in reasonable prices. Applying better AHRS would probably further reduce roll and pitch motion. Integrating such a device within the system is going to be carried out shortly. Generally the tests proved that the main goal of the project was achieved. Some problems are still present; however, the ways of improving the system have been established and changes in the system mechanics and control are going to be applied in the future.

\section{ACKNOWLEDGEMENTS}

This work was supported by Polish Agency for Enterprise Development (PARP) within the project "Ultralight Stabilized Platform for Gyrocopters” („Ultralekka Stablizowana Platforma Pomiarowa dla Wiatrakowców") under contract number: UDAPOIG.01.04.00-12-127/11-00.

\section{REFERENCES}

Adamek A., 2014. Analysis of the Photogrammetric Data Obtained From Ultralight Measuring Platform Mounted On Gyrocopter, 14th International Multidisciplinary Scientific GeoConference SGEM 2014, Conference Proceedings, Book 2, Vol. 3, pp. 11-18.

Gough V.E., Whitehall S.G., 1962. Universal tyre test machine, Proceedings of FISISTA Ninth International Technical Congress.

Kolecki J., Prochaska M., Piątek P., Baranowski J., Kurczyński Z., 2015. Stabilization of the Photogrammetric System for a Gyrocopter in Terms of the LiDAR Data Quality. In: Archives of Photogrammetry, Cartography and Remote Sensing, Vol. 27, pp. 71-82.

Kurczyński Z., Bakuła K., 2013. Generation of countrywide reference digital terrain model from airborne laser scanning in ISOK project. In: Archives of Photogrammetry, Cartography and Remote Sensing, Special Issue: Geodetic Survey Technologies, pp. 59-68.

Miraliakbari, A., Hahn, M., Engels, J., 2010. Development of a Low-cost Sensor System for Use on Gyrocopters., In: International Archives of the Photogrammetry, Remote Sensing and Spatial Information Sciences, Canadian Geomatics Conference 2010 and ISPRS Com. I Symposium, Vol. XXXVIII, No. Part 1, p. 7

Miraliakbari A., Hahn M., Engels J., 2012. Vibrations of a Gyrocopter - an Analysis Using IMUs. In: The International Archives of the Photogrammetry, Remote Sensing and Spatial Information Sciences, Melbourne, Australia, Vol. XXXIX, Part B1, pp. $497-502$.

MSWiA, 2011. Regulation of Ministry of Interior and Administration concerning data bases of aerial and satellite imageries and orthophotomap and digital terrain model (Rozporządzenie Ministra Spraw Wewnętrznych i Administracji w sprawie baz danych dotyczących zobrazowań lotniczych i satelitarnych oraz ortofotomapy i numerycznego modelu terenu). In: Dziennik Ustaw (Journal of Laws of the Republic of Poland), Vol. 263, Poz. 1571, pp. 15307-15394.

Petrie, G., 2014. SPAR Europe \& European Lidar Mapping Forum 2013. GeoInformatics, 17(1), pp. 36 - 42

Riegl, 2013, Riegl-VQ580 Technical Data.

Stewart D., 1965-66. A platform with six degrees of freedom, Proccedings of the IMechE, Vol.180, Pt. 1, No 15.

Stranneby D., 2001. Cyfrowe przetwarzanie sygnatów (Digital signal processing), BTC, Warsaw.

Zieliński T. P., 2005. Cyfrowe przetwarzanie sygnałów. Od teorii do zastosowań (Digital signal processing. From theory to practice), Wydawnictwa Komunikacji i Łączności, Warsaw. 